\title{
PATRONES DE CONOCIMIENTO DE CARPER Y EXPRESIÓN EN EL CUIDADO DE ENFERMERÍA: ESTUDIO DE REVISIÓN
}

\author{
CARPER KNOWLEDGE PATTERNS AND EXPRESSION IN NURSING CARE: REVIEW \\ STUDY
}

MODELOS DE CONHECIMENTO DA CARPER E EXPRESSÃO NO CUIDADO DE ENFERMAGEM: ESTUDO DE REVISÃO

\section{Blanca Escobar-Castellanos}

Departamento de Enfermería, Universidad de Sonora. Sonora, México. blanca.escobar@unison.mx

ORCID: 0000-0001-9239-0461

\section{Olivia Sanhueza-Alvarado}

Facultad de Enfermería, Universidad de Concepción. Concepción, Chile. ORCID 0000-0002-0184-8957

DOI: https://doi.org/10.22235/ech.v7i1.1540

Recibido: 04/10/2017

Aceptado: 15/03/2018

\begin{abstract}
Resumen: El objetivo del artículo es describir los patrones del conocimiento en enfermería de Barbara Carper y su influencia en los diferentes contextos de la disciplina de enfermería, en la investigación, educación y práctica. Metodología: revisión de la literatura científica realizada en las bases de datos de Bvs-BIREME, SciELO y PubMed durante los años 2008 al 2015, seleccionando nueve artículos que emplearon el abordaje de patrones del conocimiento de Carper como criterio de inclusión, tanto en investigaciones cualitativas como cuantitativas. La necesidad de fundamentar el quehacer profesional de Enfermería ha permitido desarrollar aspectos propios de la misma a través de valores, estructuras, formas, comportamientos y patrones de conocimiento que sirvan como directrices para ejemplificar características y modos de pensar acerca de los fenómenos de estudio. Hablar de patrones de conocimiento en la disciplina es hacer referencia a Bárbara Carper, quien identificó cuatro patrones fundamentales. Estos patrones se distinguen según el tipo de significado lógico: el empírico hace referencia a la ciencia de enfermería; el estético al arte de enfermería; el personal al conocimiento de uno mismo y los otros, y el ético permite el desarrollo del conocimiento moral en enfermería. Se concluye que cada patrón del conocimiento de enfermería tiene dimensiones y elementos esenciales que permiten su comprensión; todos son indivisibles, se pueden emplear de forma individual, pero se integran como un todo cuando se desarrolla la práctica del cuidado.
\end{abstract}

Palabras clave: Enfermería, Cuidados de Enfermería, Educación en Enfermería. 


\begin{abstract}
The goal of this article is to describe Barbara Carper's nursing knowledge patterns and their influence in the different contexts of the nursing discipline, in research, education and practice. Methodology: review of the scientific literature found in the databases Bvs-BIREME, SciELO and PubMed during the years 2008 to 2015, selecting nine articles that used the approach of Carper's knowledge patterns as an inclusion criterion, both in qualitative and quantitative research. The need to give a solid base to the Nursing professional work allowed to develop patterns through values, structures, forms and behaviors, knowledge patterns that serve as guidelines to exemplify characteristics and ways of thinking about the topics of study. Speaking of knowledge patterns in this discipline is to necessarily refer to Barbara Carper, who identified four fundamental knowledge patterns. These patterns are distinguished according to the type of logical meaning: the empirical one refers to the science of nursing; the aesthetic one to the art of nursing; the personnel one to the knowledge of oneself and others; and the ethical one allows the development of moral knowledge in nursing. It was concluded that each nursing knowledge pattern has essential dimensions and elements that make easier its understanding. They are all indivisible; they can be used individually, but they are integrated as a whole, when the practice of care is developed.
\end{abstract}

Key words: Nursing, Nursing Care, Nursing Education,

Resumo: Objetivo: Descrever os padrões de conhecimento de enfermagem de Barbara Carper e sua influência nos diferentes contextos da disciplina de enfermagem, em pesquisa, educação e prática. Metodologia: revisão da literatura científica feita nas bases de dados da Bvs-BIREME, SciELO e PubMed, durante os anos de 2008 a 2015, selecionando nove artigos que utilizaram a abordagem dos padrões de conhecimento de Carper como critério de inclusão, tanto na pesquisa qualitativa e quantitativa. Desenvolvimento: a necessidade de basear o trabalho profissional de Enfermagem permitiu desenvolver aspectos da enfermagem através de valores, estruturas, formas, comportamentos e padrões de conhecimento que servem como diretrizes, para exemplificar características e formas de pensar sobre os fenômenos de estudo Falar sobre padrões de conhecimento na disciplina é referir Barbara Carper que identificou quatro padrões de conhecimento fundamentais. Esses padrões são distinguidos de acordo com o tipo de significado lógico: o empírico se refere à ciência da enfermagem; a estética do art of nursing; o pessoal para o conhecimento de si mesmo e para os outros e o ético permite o desenvolvimento do conhecimento moral na enfermagem. Conclusão: Cada padrão de conhecimento de enfermagem tem dimensões e elementos essenciais que permitem sua compreensão, todas eles são indivisíveis, podem ser usados individualmente, mas são integrados como um todo, quando a prática do cuidado é desenvolvida.

Palavras-chave: Enfermagem, Enfermagem, Educação em Enfermagem 


\section{INTRODUCCIÓN}

La Enfermería ha sido considerada como ciencia sólo a partir de los últimos decenios, en donde su desarrollo teórico se ha potenciado a través del estudio científico de los fenómenos que integran el cuidado de la salud humana. Esto ha permitido que se constituya en una ciencia humanística y social con un cuerpo de conocimientos propio (1). Su desarrollo como disciplina científica permite integrar fundamentos teóricos (Ciencia de Enfermería) y práctica profesional (dominio específico que la diferencia de otras profesiones), ambos delimitando un campo de estudio, de saberes e interpretaciones (2-3).

La integración de la ciencia y la profesión permiten a la enfermera promover el cuidado humano en diferentes momentos del proceso vital y en diferentes contextos, considerando a la persona con una visión holística del ser, focalizado en las respuestas humanas frente a problemas de salud reales o potenciales que demandan intervenciones de enfermería humanísticas y de calidad. La necesidad de fundamentar el quehacer profesional ha permitido desarrollar aspectos propios de la enfermería a través de valores, estructuras, formas, comportamientos y patrones de conocimientos que sirven como directrices para ejemplificar características y modos de pensar acerca de los fenómenos de estudio. La comprensión, sobre todo, de los patrones de conocimiento que emplea enfermería es esencial para su enseñanza y aprendizaje, lo cual permite identificar la variabilidad de aspectos que competen a esta disciplina y qué clase de conocimientos son de mayor valor en la misma (4).

Referirse a patrones de conocimiento en esta disciplina es hacer referencia a Barbara Carper, quien plantea "cuatro patrones de conocimiento fundamentales que pueden ser identificados a través del análisis de conceptos y de la estructura sintáctica del conocimiento de enfermería"; estos patrones se distinguen según el tipo de significado lógico: el empírico hace referencia a la ciencia de enfermería; el estético al arte de enfermería; el personal al conocimiento de uno mismo y los otros; y el ético permite el desarrollo del conocimiento moral en enfermería (4).

Durán de Villalobos, al hacer referencia a éstos, señaló que "los patrones del conocimiento de enfermería son elementos indispensables de fundamentación para el desarrollo del conocimiento y la práctica; se expresan de manera integrada, existiendo entre ellos conexiones que permiten la expresión del cuidado y de las intervenciones de enfermería de forma holística" (5). El presente trabajo tiene como objetivo describir los patrones del conocimiento en enfermería de Barbara Carper y su influencia en los diferentes contextos de esta disciplina en la investigación, la educación y la práctica.

\section{METODOLOGÍA}

Se llevó a cabo una revisión de la literatura científica realizada en las bases de datos de Bvs-BIREME, SciELO y PubMed durante los años 2008 al 2015, usando como criterio de inclusión la selección de aquellos artículos que emplearan el abordaje de los patrones del conocimiento de Barbara Carper en situaciones de cuidado de enfermería, tanto en investigaciones de tipo cualitativas como cuantitativas. Se seleccionaron cinco artículos originales de tipo cualitativo, dos artículos de tipo cuantitativo y dos artículos de revisión y 
análisis que establecen propuestas para desarrollar los patrones de conocimiento al brindar el cuidado de enfermería.

\section{Patrones de Conocimiento}

El abordaje de los términos "patrón” y "conocimiento", según la Real Academia Española, permite entender la conjugación de ambos vocablos. "Patrón" hace referencia a un modelo que sirve de muestra para "sacar otra cosa igual"; en cambio el "conocimiento" permite conocer o tener noción de algo y el conocer (del latín Cognoscěre) indica "averiguar por el ejercicio de las facultades intelectuales, la naturaleza, cualidades y relaciones de las cosas" (6).

Devlin en 1999 mencionó que el conocimiento "no es un objeto"; requiere "un conocedor". Considera que es una actividad intrínsecamente humana, que permite a la persona obtener información para utilizarla con un propósito definido; además contiene creencias, valores, compromisos, experiencia, información contextual, percepciones de expertos e intuición; por lo tanto se convierte en una acción deliberada que trata significados, dependiendo de los contextos específicos donde se obtenga o se produzca (7). Según la jerarquía del conocimiento (Bende y Fish, 2000), cuando el individuo realiza el procesamiento de la información, el conocimiento se concibe como un flujo continuo que pasa desde la obtención de datos (a través de documentos o bases de datos) hasta la comprensión de éstos con un propósito definido, transformándose en conocimiento. La figura 1 muestra la jerarquía del conocimiento (7).

Para la disciplina de enfermería esta jerarquía del conocimiento es relevante porque involucra "un acto consciente, una forma particular e individual de visualizar las complejidades de una situación y de reunir recursos internos experimentados anteriormente para darle significado", lo que representa la puesta en práctica de habilidades, destrezas y conocimientos para brindar un cuidado de enfermería integral, de forma consciente y deliberada, particularizando cada situación como una experiencia de cuidado única para la enfermera y la persona (1).

\section{Patrones de Conocimiento de Enfermería de Barbara Carper (1978)}

Barbara Carper en 1978 describió lo que llamó "patrones fundamentales del conocimiento de enfermería", expresando que "el cuerpo de conocimientos que da soporte a la enfermería se manifiesta por patrones, o sea, por medio de formas características de expresión externa e interna que dejan ver la manera como se piensa sobre un fenómeno" (5). Estos patrones fueron publicados en la primera edición de Advances in Nursing Science, basado en su trabajo doctoral, en que creó una tipología de patrones del conocimiento en enfermería a los cuales denominó: empírico/la ciencia de enfermería; estético/arte de enfermería, el 
componente del conocimiento personal en enfermería; y ético/el componente del conocimiento moral en enfermería (8).

La introducción de esta tipología permitió de alguna manera organizar, clarificar y explorar las diversas situaciones donde enfermería desarrolla su práctica de cuidado. Cada patrón de conocimiento hace aportes significativos a esta disciplina en áreas específicas de su desarrollo. Durán de Villalobos señaló que están entrelazados y son indivisibles, que permiten centrar el desarrollo disciplinar al guiar su práctica y que surgieron del análisis y estructura del conocimiento de enfermería. Además mencionó que "cada patrón es diferente al todo de enfermería, pero a su vez contribuye al conocimiento total y es igualmente importante" (9).

Los patrones de conocimiento de Carper, desde sus inicios, fueron integrados a la enseñanza y la práctica de enfermería por considerarse elementos esenciales para evidenciar el conocimiento de la disciplina, porque cada patrón representa una forma de conocimiento con posibilidades de ser entendibles, comprendidos y susceptibles de credibilidad (9). Además, aumentan la complejidad y la diversidad epistemológica de la enfermería (10). Es importante que todos los patrones de conocimiento deban integrarse en su conjunto, para favorecer la toma de decisiones clínicas de enfermería de forma deliberada y racionalizada; el hecho de no integrarlos en su conjunto puede impedir elecciones efectivas y producir resultados negativos en la atención que se otorga (11).

\section{Descripción de los Patrones del Conocimiento de Carper}

El patrón empírico fue designado como "la ciencia de enfermería"; hace referencia al empleo de leyes generales y teorías con el propósito de "describir, explicar y predecir fenómenos de especial preocupación para la disciplina, entrega evidencia empírica que puede ser empleada para la organización y clasificación del conocimiento en enfermería" (4). El avance en teorías científicas y modelos conceptuales ha permitido el desarrollo de la ciencia de enfermería y de la práctica, los cambios paradigmáticos, la explicación y predicción de hechos que permiten nuevas perspectivas para abordar los fenómenos familiares de salud y enfermedad en relación con el proceso de la vida humana (12). La literatura permite afirmar que el patrón empírico ha sido el que más evidencia objetiva y tangible ha aportado a la enfermería; brinda el sustento teórico para seguir desarrollando el conocimiento de la misma y permite el desarrollo de la investigación en diversos campos de interés; sus resultados aportan una gama de elementos teóricos y prácticos que permiten fundamentar la práctica profesional, para mejorar la calidad del cuidado que se brinda. En relación a este patrón Jacobs y Chinn mencionaron que "el conocimiento empírico representa el conocimiento que se acumula a través de la experiencia sensorial, comprende conocimientos adquiridos y transmitidos a través del entendimiento" (11). Sugieren preguntas como: ¿Qué es lo que representa? y ¿Cómo se hace ésto representativo? Las respuestas permiten dar sustento teórico metodológico al fundamentar la práctica profesional, al implementar teorías y modelos propios de enfermería en base a la problemática que se desea abordar, desarrollando propuestas para mejorar el cuidado de enfermería y aportar hallazgos para evidenciar el desarrollo disciplinar (11). 
El patrón estético o "arte de enfermería" no se origina como resultado de la investigación empírica. Carper argumentó que "por lo tanto puede haber reticencia consciente para incluirlo como un saber de enfermería, podría describirse como una admisión tácita de que enfermería en parte, es al menos un arte". Además mencionó que "no se había hecho esfuerzo suficiente para elaborar o hacer explícito este conocimiento, donde muchos asocian el arte como una categoría general de habilidades técnicas y manuales desarrolladas en la práctica de enfermería" (4). Una experiencia estética involucra la creación y/o apreciación de expresiones singulares o particulares de posibilidades subjetivas imaginadas o reales que "se originan de una proyección discursiva del lenguaje" (4). En este sentido es necesario desarrollar habilidades de comunicación, ser creativos, empatizar y establecer una relación terapéutica humanizada con el paciente/familia, para identificar aspectos objetivos o subjetivos que permiten detectar las necesidades reales de la persona que transita por una experiencia de salud-enfermedad, para poder crear un entorno que favorezca su recuperación (13). El papel principal de la enfermera es actuar como un ser humano sensible y proactivo, que la diferencie de los demás profesionales de la salud, porque en su actuar profesional se reflejará "el arte", presente en todos los aspectos de la práctica de enfermería $(5,14)$. Desarrollar la creación estética en enfermería es complejo, requiere abstracción, la que se hace visible a través de las interacciones, las intervenciones del cuidado y las actitudes que desarrolla la enfermería en respuesta a las demás personas; permite la transformación del encuentro inmediato entre paciente-enfermera en una percepción significativa en sí misma, le adjudica significado y se refleja en la acción tomada por la enfermera $(7,15)$. La estética en el cuidado implica no sólo aplicar conocimiento científico, sino evidenciar que se adoptan medidas prioritarias e indispensables para que el paciente se recupere o desarrolle la capacidad de hacer frente a la situación; este cambio permitirá el bienestar del paciente y será el reflejo de las acciones emprendidas por la enfermera.

La empatía es un modo importante en el patrón de conocimiento estético; ponerla en práctica permite conocer a la otra persona en situaciones singulares y particulares; "es la experiencia de sentir a través del conocimiento empático". Enfermería tiene muchas habilidades para percibir y empatizar con la vida de otras personas, tiene una gama de conocimientos que le permiten identificar y entender diversas formas de percibir la realidad para proveer y satisfacer de forma efectiva los cuidados que provee. A partir de lo anteriormente expuesto es importante que la enfermera se plantee el siguiente cuestionamiento: ¿Sé lo que hago? y ¿Hago lo que sé?. En base a las respuestas la enfermera desarrollará el arte en su actuación profesional de la forma más asertivamente posible (11).

El patrón de conocimiento personal expresa el conocimiento de sí mismo (en relación con los demás); desde la perspectiva intrínseca se considera fundamental, requiere el desarrollo del "conocimiento personal"; por eso es el más problemático para dominar y enseñar pero a la vez es el más esencial para entender "el significado de la salud en términos de bienestar individual" $(11,16)$. Para enfermería el proceso interpersonal que involucra la interacción, relaciones e intercambios entre la enfermera y el paciente es indispensable; esto se logra a 
través de conocerse uno mismo y entender a los otros para establecer relaciones terapéuticas que favorezcan la salud del individuo $(4,11)$. La complejidad de desarrollar y evidenciar este patrón de conocimiento es debido a que involucra la "experiencia del ser", que se obtiene al desarrollar realidades y potencialidades personales facilitando los procesos de experimentar, encontrarse y enfocarse, los cuales involucran el continuo conocimiento de sí mismo. Si la enfermera no se conoce a sí misma, será difícil entablar una relación con otro ser humano, entenderlo o confrontarlo como persona, estableciéndose barreras en la relación de cuidado que se otorga. Es importante desarrollar la autenticidad como elemento indispensable en la relación con los otros, lo que implica revelar el "ser personal" (lo que uno es en realidad, que es conocido en privado) en cualquier momento o situación; mientras que el "ser externo", es el más comúnmente demostrado y fácilmente puede ser revelado a otros. Pero no precisamente significa que las relaciones de interacción son de autenticidad. Jacobs y Chinn expresaron que este patrón demuestra el "ser auténticos a través del desarrollo del ser" (11). Desarrollar esta potencialidad se logra a través de una vida interior rica y plena; es prioritario que enfermería pueda sensibilizarse a través del uso de las artes, poesía, literatura, narración de cuentos y todas aquellas técnicas que permitan realizar un esfuerzo por comprender al ser humano (persona) (8). Es importante que enfermería se pregunte ¿Sé lo que hago? y ¿Hago lo que sé?, lo cual permite analizar lo que realmente se hace en el ámbito de la práctica al identificar el verdadero ser, al actuar de forma auténtica o no y permitir una reflexión congruente con nuestro desempeño profesional (11).

El patrón de conocimiento ético es el componente moral de la disciplina. Permite demostrar el deber ser de la disciplina fundamentado en valores humanísticos personales, profesionales e institucionales (17). Además establece el compromiso y respeto a la vida humana, sustenta la toma de decisión personal en situaciones difíciles y complejas del moderno mundo del cuidado a la salud, permite elecciones al cuestionarse acerca de los derechos morales y errores en las acciones vinculadas con el cuidado, el tratamiento de las enfermedades y la promoción de la salud $(4,15)$. Para normar la conducta ética se cuenta con los códigos morales y éticos propios de la profesión, los cuales permiten guiar el actuar ético profesional de las enfermeras, basado en principios primarios obligatorios de preservar la vida, aliviar el sufrimiento, promover la salud; pero éstos no incluyen respuestas en temas morales de difícil decisión personal que pueden presentarse en la enseñanza y la práctica de enfermería. Estas situaciones hoy en día son muy comunes en los sistemas de salud; enfermería tiene que enfrentarse a estos hechos, haciendo uso y aplicación de principios y el deber ser de la disciplina (15). Este patrón debe evidenciar que la actuación de la enfermera esté apegada a una conducta moralmente aceptada, adicionando elementos como la virtud, la empatía y la compasión al tomar elecciones individuales de forma consciente y deliberada, sustentada por el conocimiento empírico que todo profesional debe demostrar como individuo, con sentimientos de totalidad e integridad, lo cual es explicado desde el punto de vista epistemológico (lo que se conoce que debe hacer la enfermera) y ontológico (lo moralmente aceptado) (10). El patrón ético en enfermería implica entender las diferentes posiciones filosóficas, para responder a las 
preguntas de Jacobs y Chinn ¿Qué es bueno? ¿Qué es lo correcto? ¿Qué es lo justo? en las situaciones de cuidado que implementa en el paciente o al enfrentarse a dilemas éticos o bioéticos y morales $(4,5,11)$. Permite a la enfermera establecer relaciones terapéuticas de respeto, ejecutar intervenciones de enfermería basadas en un consentimiento informado al considerar a la persona en su integralidad y totalidad, como sujeto inalienable, con derechos como persona y como paciente. Además, Martínez señaló que se debe tener la capacidad de mostrar sensibilidad en situaciones particulares, establecer vínculos con la familia y el equipo de salud para gestionar el cuidado, demostrar un compromiso hacia la profesión, actuar con respeto, tolerancia, solidaridad, promover la autonomía, la justicia y sobre todo proteger al paciente al no causar daño alguno $(14,18)$.

Posteriormente a la publicación de estos patrones del conocimiento de enfermería, diez años después Jacobs y Chinn, en 1988, publicaron un modelo de conocimiento de enfermería tomando como base los cuatro patrones originalmente nombrados por Carper, consistentes en una propuesta para presentar las dimensiones que se encontraron presentes en cada patrón del conocimiento, lo cual permitió su mejor comprensión. Son éstos: a) la creativa, que permite capturar cómo se genera el conocimiento, b) la expresiva, que captura cómo el patrón es demostrado y reconocido y c) la evaluación, que permite realizar una examinación del conocimiento por medio de preguntas críticas claves, dentro de un contexto de desarrollo y utilizar índices de credibilidad para comprobar el conocimiento desarrollado. La tabla 1 muestra las dimensiones del Modelo de Conocimiento de Enfermería propuesto (11).

Tabla 1. Dimensiones del Modelo de Conocimiento de Enfermería

\begin{tabular}{|c|l|}
\hline DIMENSIONES & \multicolumn{1}{c|}{ CARACTERÍSTICAS } \\
\hline Creativa & $\begin{array}{l}\text { Captura cómo es generado el conocimiento y extendido a través } \\
\text { del uso. Implica la interacción del proceso del producto, implica } \\
\text { movimiento. }\end{array}$ \\
\hline Expresiva & $\begin{array}{l}\text { Captura cómo el patrón de conocimiento es demostrado y } \\
\text { reconocido. }\end{array}$ \\
\hline Evaluación & $\begin{array}{l}\text { Proporciona un examen de conocimientos a través de: } \\
\text { 1. Hacer preguntas críticas de las formas de conocimiento } \\
\text { 2. Dentro de un contexto de proceso } \\
\text { 3. Usar un patrón específico de índice de credibilidad }\end{array}$ \\
\hline
\end{tabular}

Fuente: Jacobs-Kramer M, Chinn P. Perspectives on knowing: A Model of Nursing Knowledge (1988).

En la tabla 2 se aprecia lo que Jacobs y Chinn incluyeron como elementos esenciales del Modelo de Conocimiento de Enfermería, para cada patrón de Carper, según sus tres dimensiones $(8,11)$. 
Tabla 2. Elementos del Modelo de Conocimiento de Enfermería por Dimensión

\begin{tabular}{|c|c|c|c|c|c|}
\hline \multicolumn{2}{|c|}{ DIMENSIÓN } & EMPÍRICO & ÉTICO & PERSONAL & ESTÉTICO \\
\hline \multicolumn{2}{|c|}{ Creativa } & $\begin{array}{l}\text { Describir } \\
\text { Explicar } \\
\text { Predecir }\end{array}$ & $\begin{array}{l}\text { Valorar } \\
\text { Clarificar } \\
\text { Defender }\end{array}$ & $\begin{array}{c}\text { Encontrarse } \\
\text { Enfocarse } \\
\text { Darse cuenta } \\
\text { de. }\end{array}$ & $\begin{array}{c}\text { Tomar } \\
\text { parte de. } \\
\text { Interpretar } \\
\text { Previsor }\end{array}$ \\
\hline \multicolumn{2}{|c|}{ Expresiva } & $\begin{array}{c}\text { Datos } \\
\text { Teorías } \\
\text { Modelos } \\
\text { Descripciones } \\
\text { para impartir } \\
\text { comprensión }\end{array}$ & $\begin{array}{c}\text { Códigos } \\
\text { Normas } \\
\text { Teorías éticas } \\
\text { normativas } \\
\text { Hacer } \\
\text { descripciones } \\
\text { de decisiones } \\
\text { éticas }\end{array}$ & $\begin{array}{c}\text { Yo: Auténtico } \\
\text { y revelador }\end{array}$ & Arte-Acto \\
\hline \multirow{3}{*}{$\begin{array}{l}\text { Evalua } \\
\text { ción }\end{array}$} & $\begin{array}{l}\text { Pregunta } \\
\text { Crítica }\end{array}$ & $\begin{array}{l}\text { ¿Qué es lo que } \\
\text { representa? } \\
\text { ¿Cómo es } \\
\text { representativo? }\end{array}$ & $\begin{array}{c}\text { ¿Es correcto? } \\
\text { ¿Es justo? }\end{array}$ & $\begin{array}{c}\text { ¿Sé lo que } \\
\text { hago? } \\
\text { ¿Hago lo que } \\
\text { sé? }\end{array}$ & $\begin{array}{c}\text { ¿Qué } \\
\text { significa } \\
\text { esto? }\end{array}$ \\
\hline & $\begin{array}{l}\text { Contexto/ } \\
\text { Proceso }\end{array}$ & Replicación & Diálogo & $\begin{array}{c}\text { Respuesta y } \\
\text { reflexiona }\end{array}$ & Critica \\
\hline & $\begin{array}{l}\text { Índice } \\
\text { Credibilidad }\end{array}$ & Validación & Justicia & Congruencia & $\begin{array}{l}\text { Significado } \\
\text { consensual }\end{array}$ \\
\hline
\end{tabular}

Fuente: Jacobs-Kramer M, Chinn P. Perspectives on knowing: A Model of Nursing Knowledge (1988)

El modelo propuesto por Jacobs y Chinn ha permitido identificar los elementos que se consideran indispensables y que deben estar presentes al desarrollar cada patrón de conocimiento de enfermería, facilitando la comprensión de cada uno y a la vez permitiendo analizar si cada patrón se está desarrollando de forma adecuada al poder evaluarlo.

Otro patrón incorporado a los conocimientos de enfermería ya existentes fue el desarrollado por White en 1995, denominado patrón sociopolítico. Considerado el quinto patrón de conocimiento, permitió conocer el contexto sociopolítico del paciente y el contexto sociopolítico de la enfermera, al considerar los ambientes donde se desarrollan las personas y sus interacciones, siendo esencial para entenderlos en su totalidad. Este patrón permite entender la importancia de enfermería en la sociedad y viceversa, sin dejar de lado las políticas establecidas socialmente $(8,17)$. White mencionó que los otros patrones responden a las preguntas: ¿Quién?, ¿Cómo? y ¿Qué? de la práctica de enfermería y el patrón sociopolítico responde a ¿Dónde?, dando respuesta a cuál es la situación donde la enfermera establece relaciones con el paciente, abordando el contexto donde la enfermera y el cuidado de la salud tienen lugar (8). 
Por otro lado, la adición del patrón de conocimiento emancipatorio por Jacobs y Chinn permitió conocer el contexto social y político de la práctica de enfermería y del cuidado a la salud, al reconocer que existen serias barreras sociales que afectan la salud y el bienestar de las personas. Este patrón destaca la capacidad no sólo de hacer evidentes las injusticias de orden social; se requiere conocimiento para examinar críticamente las situaciones, porque las injusticias no son evidenciadas. Es necesario que se aborden a través de la reflexión crítica y la acción; el proceso de conocimiento emancipador se desarrolla a través de la práctica (15).

La tipología de los patrones de conocimiento de enfermería desarrollados anteriormente dan sustento a la disciplina de enfermería. Cada uno puede ser abordado de forma individual, pero todos ellos se deben reflejar en el actuar profesional porque están entrelazados y son indivisibles. Carper mencionó que "los patrones del conocimiento se pueden enunciar como una cultura o una filosofía intelectual de enfermería, pero no integran una teoría”, y permiten responder a la pregunta ¿Qué es enfermería? y ¿Qué es la práctica de enfermería? (12).

\section{RESULTADOS}

Los resultados de la evidencia científica con abordaje de los patrones del conocimiento de Carper en la educación, práctica clínica y la investigación en enfermería fueron los descritos a continuación.

En la revisión de la literatura científica realizada se encontró que la tipología sustenta los marcos de referencia de varias investigaciones fundamentando la epistemología y ontología, los cuales han sido citados más de 1800 veces en la literatura científica por su gran influencia para la enfermería (19). Al respecto, Laura Alberto argumentó que la práctica que genera la investigación desarrolla el patrón empírico, "aunque otros autores podrían argumentar lo contrario"; sostiene que los patrones de conocimiento ético, estético y personal pueden ser sujetos de ser investigados (20). La mayoría de la literatura revisada, que pretende evidenciar el desarrollo de los patrones del conocimiento en la educación o la asistencia, desarrolla la investigación de tipo cualitativo al indagar situaciones de cuidado de tipo fenomenológico, empleando como técnica la narrativa al explorar las experiencias de la persona al brindar el cuidado. Fawcett y Lee reconocieron la importancia de desarrollar la producción del conocimiento en enfermería desde la formación de pregrado, maestría y doctorado, para evidenciarlo en la práctica de enfermería; es importante involucrar a todo el personal de enfermería, docentes e investigadores de enfermería para generar nuevas teorías y evaluar las existentes; mencionan la importancia de no sólo desarrollar la ciencia de enfermería, sino de incorporar todas las áreas específicas de la misma, debido a que el conocimiento necesita de la teoría basada en la evidencia práctica, pero se tienen que considerar los patrones de conocimientos de Carper y el patrón sociopolítico debido a que el resultado podría generar la producción de nuevas teorías derivadas de cada patrón (21). 
En la educación, algunos investigadores han empleado la narrativa como técnica de investigación para evidenciar los patrones del conocimiento en diversos ámbitos de aprendizaje. Gómez et al midieron la percepción del cuidado en estudiantes de primer semestre empleando instrumentos que abordaban algunos patrones de conocimiento, sustentados en teorías de enfermería. Se encontró que el patrón personal y estético fueron percibidos con más baja frecuencia (22). Landeros empleó "la narrativa en un incidente crítico ocurrido en la atención a una paciente, en estudiantes de pregrado, con la finalidad de reflexionar sobre la situación a las cuales el estudiante se enfrenta en su práctica clínica" (23).

Por otro lado, Bautista midió el conocimiento e importancia en los estudiantes de enfermería sobre el consentimiento informado en actos del cuidado de enfermería, identificando que el patrón estético obtuvo variaciones altas, reflejando aspectos que guardaban relación con la función del diseño del cuidado partiendo del bagaje cultural que el estudiante le aporte, logrando su máxima expresión a lo largo de su ejercicio profesional e imprimiendo su sello individual y diferenciador (13). Muñoz, Morales y Torres expusieron la utilización de material cinematográfico en una actividad de formación educativa para mejorar la implementación de la metodología del cuidado, al ilustrar aspectos relacionados con la experiencia de la enfermedad, las relaciones con los profesionales de la salud y la imagen de los mismos en la filmografía existente. Junto con lo anterior, también mencionaron que el cine es un recurso inestimable en la educación por su capacidad de aprehender la experiencia humana como ninguna otra manifestación artística (24).

En el área de la educación en enfermería se pudo evidenciar que en pleno siglo XXI la educación en esta disciplina ha tenido una gran transformación debido al desarrollo teórico que la sustenta. A nivel educativo es prioritario considerar a los estudiantes en el tipo de aprendizaje que adquieren. La incorporación de los patrones del conocimiento forma parte de la mayoría de los programas de enfermería, porque permite de alguna forma sustentar la epistemología de la disciplina; por lo tanto es casi un requisito indispensable su enseñanza en la formación de pregrado (9). Para incentivar el desarrollo de habilidades y destrezas en la formación de pregrado es necesario establecer planes de estudio que involucren al estudiante y al profesor con la finalidad de generar la capacidad auto-reflexiva, crítica y analítica del estudiante; para favorecer el desarrollo intelectual, la apreciación de las artes, las habilidades orales y escritas, la toma de decisiones, la adquisición de valores, la solución de problemas, la introspección y la incursión en la investigación desde su formación básica (25). Se hace evidente la importancia de incorporar las artes y las humanidades en la educación de enfermería y el uso de un enfoque integrado con sus patrones de conocimiento (10). Para que el estudiante desarrolle una mayor percepción sensorial es necesario incentivar el desarrollo de la capacidad creativa (imaginación/visualización) no solamente para las actividades o procedimientos técnicos, sino para la interacción con las demás personas. Se debe motivar el desarrollo de la dimensión cognitiva de otras áreas de interés (histórico, lingüístico, técnico o cultural) que 
favorezca la adquisición de conocimiento de forma globalizada y un bagaje para mejorar su expresión verbal y corporal. Es necesario potenciar la dimensión expresiva para favorecer el patrón personal y estético, que aprenda a exteriorizar sus sentimientos, deseos y emociones a través de diversas técnicas que componen el arte (los sentimientos se materializan a través de la pintura, expresión corporal, dramatización, poesía, expresión literaria, música, grabación, la filmación de sus experiencias, el uso de la tecnología de vanguardia) entre otras. Se debe incentivar el desarrollo del patrón de conocimiento ético al establecer estrategias de aprendizaje que le permita analizar situaciones clínicas que lo lleven a experimentar la toma de elecciones de tipo ético-morales.

En la práctica clínica del cuidado de enfermería la contribución muestra que existe suficiente sustento científico de que esta disciplina emplea los patrones del conocimiento para evidenciar el desarrollo del cuidado en diversas áreas asistenciales. Muñoz y Casique narran una experiencia de cuidado en la relación enfermera-paciente (niño con secuelas neurológicas y motoras) que involucró el sentimiento de "ternura"; evidenciaron que los patrones de Carper son una herramienta de gran importancia en el desarrollo del conocimiento disciplinar con su enfoque epistemológico y ontológico pues permiten estudiar el saber de la enfermera como persona (26). Por otra parte, Gómez-Palencia analizó la técnica narrativa de enfermería en un paciente adulto con enfermedad cardiovascular en el servicio de urgencias, que fue atendido por estudiantes de enfermería y su docente. Empleó los patrones de conocimientos al describir la experiencia del cuidado de enfermería para identificar el fenómeno de salud de la persona enferma, lo cual facilitó que el personal de enfermería cumpliera con su deber de brindar el cuidado de forma holística (27). Por su parte Briñez analizó una narrativa de enfermería derivada de una entrevista en el hogar a la cuidadora de una persona con enfermedad crónica degenerativa; empleó la teoría de cuidados de Kristen Swanson y evaluó seis patrones del conocimiento: personal, empírico, ético, estético, sociopolítico y emancipatorio, destacando que las enfermeras requieren desarrollar los valores éticos y estéticos como habilidades para brindar el arte de cuidado que es "el escuchar" (10). Pech empleó la narración para analizar los patrones de conocimiento a través de la comunicación y establecer una relación de afecto entre una persona de la tercera edad y la enfermera, evidenciando que el afecto que demuestra la enfermera a las personas a su cuidado es de suma importancia y trascendental para su mejoría (28). Así también Hernández realizó una narrativa de la experiencia de cuidado en un paciente adulto mayor crónico con dificultades para la comunicación e identificó que los valores éticos y estéticos deben ser desarrollados por las enfermeras para poder desarrollar el arte de cuidar como es "el escuchar" (17). Por eso el conocimiento estético es un elemento vital para la práctica de enfermería, pero lamentablemente es percibido como imaginario dentro de la profesión; cada vez es más distante del significado social de la enfermería. En ocasiones el arte de enfermería es relacionado con la búsqueda de la identidad profesional o la búsqueda de una práctica integradora al desarrollar más la técnica (hacer las cosas bien) que demostrar la sensibilidad necesaria acorde a las situaciones que se presentan con el paciente. El establecer una relación interpersonal como esencia de la 
enfermería entre sujeto - objeto, permitirá desarrollar el conocimiento personal; sin embargo, se requiere de experiencia para lograrlo (29).

En la investigación de enfermería, Espitia realizó un análisis del cuidado de enfermería en el ámbito de la cirugía plástica a partir de los patrones de conocimiento para mostrar cómo a través de éstos se expresa, desarrolla y sustenta la práctica del cuidado, resaltando como relevante que las formas de adquirir cada patrón de conocimiento van desde lo simple a lo complejo y debe ser una tarea personal que aumente la calidad y la significancia de cada acto de cuidado (18). En la Tabla 3 se muestra el resumen de los artículos seleccionados.

\section{CONCLUSIONES}

El empleo de los patrones del conocimiento de Carper en enfermería desde su propuesta ha servido de sustento filosófico para esta disciplina. Surgieron en una época muy temprana del desarrollo incipiente de la ciencia de enfermería y después de décadas siguen siendo un pilar fundamental para la epistemología (conocimiento propio de la enfermería) y la ontología (esencia de ser). La tipología de patrones del conocimiento de Carper fue una propuesta que permitió organizar y agrupar los saberes de la disciplina. Cada patrón del conocimiento de enfermería tiene dimensiones y elementos esenciales que permiten su comprensión; todos son indivisibles, se pueden emplear de forma individual, pero se integran como un todo cuando se desarrolla la práctica del cuidado. Los cuatro patrones se pueden manifestar a través de la creatividad, la expresión y pueden ser evaluados en la aplicación de los cuidados de enfermería. El patrón de más difícil evidencia, pero muy importante, es el personal, ya que es necesario que la enfermera permita conocerse primero como persona para después establecer relaciones terapéuticas auténticas entre ella y la persona (el paciente) a quien brindará el cuidado, para potenciar el bienestar del individuo.

\section{REFERENCIAS BIBLIOGRÁFICAS}

1. Rubio AM, Arias BM. Fenomenología y conocimiento disciplinar de enfermería. Revista Cubana de Enfermería. 2013; 29 (3):191-198.

2. Vílchez BV, Sanhueza AO. Enfermería: Una disciplina social. Enfermería en Costa Rica. 2011;32 (1): 81-88.

3. Argote LA, Burbano C, Santamaría C, Vásquez ML. El desarrollo disciplinar: Razón de ser, sentipensar y hacer en enfermería. Colomb Med. 2011;42 (1):78-85.

4. Carper BA. Fundamental patterns of knowing in nursing. Advances in nursing science. (1978);1(1):13-24.

5. Durán de Villalobos M. La ciencia, la ética y el arte de enfermería a partir del conocimiento personal. Aquichan. 2005;5(1):86-95.

6. Real Academia Española. Conocimiento. Diccionario de la lengua española. Disponible en: http://lema.rae.es/drae/?val=conocimiento

7. Liberona D, Ruiz M. Análisis de la implementación de programas de gestión del conocimiento en las empresas chilenas. Estudios gerenciales. 2013;29:151 
8. White J. Patterns of knowing: Review, critique and update. Advances in nursing science. (1995);17 (4):73-86.

9. Durán de Villalobos M. Enfermería: Desarrollo teórico e investigativo. Proyecto Innovar. Universidad Nacional de Colombia. 2001. 26-39.

10. Briñez AK. Narrativa de enfermería: visión y patrones de conocimiento en una entrevista en el hogar. Rev. Colombiana de Enfermería. 2014;9(9):142-148.

11. Jacobs-Kramer M, Chinn P. Perspectives on Knowing: A Model of Nursing Knowledge. Scholarly Inquiry for Nursing Practice: An International Journal. 1988;2(2).

12. Durán de Villalobos MM. Marco epistemológico de la enfermería. Aquichán. 2002;2(1):7-18.

13. Bautista EG. Conocimiento e importancia en los estudiantes de enfermería sobre el consentimiento informado en actos del cuidado de enfermería. Investig Enferm. Imagen Desarr. 2015;17(2): 1-17.

14. Hernández D. Una experiencia fundamental para mejorar el arte y la ciencia de cuidar. Revista Ciencia y cuidado. 2013;10 (1):59-66.

15. Martínez PM. Arte y ciencia de la enfermería. Rev méd electrón. 2008; 30 (1).

16. Ramírez E.N, Quintana ZM, Sanhueza AO, Valenzuela SS. El paradigma emancipatorio y su influencia sobre el desarrollo del conocimiento en enfermería. Enferm. glob. 2013:12(30):410-421.

17. Hernández DV. Una experiencia fundamental para mejorar el arte y la ciencia de cuidar. Revista Ciencia y Cuidado. 2013;10(1):59-66.

18. Espitia CL. El cuidado de enfermería en el contexto de la cirugía plástica estética: una reflexión a partir de los patrones de conocimiento. Umbral científico. 2009;15: 8-14.

19. Eisenhauer ER. An interview with Dr. Barbara A. Carper. Advances in Nursing Science. 2015;38 (2):73-82.

20. Alberto, L. Formación para la ciencia: una reflexión sobre los desafíos de la investigación en enfermería en la región de Latinoamérica. Rev. Cuidarte. 2014;5 (2):713-716.

21. Fawcett J, Lee R. Advancing Nursing Knowledge: A Response to Burns' Letter to the Editor. Nursing Science Quarterly. 2014; 27 (1):88-90.

22. Gómez RO, Daza CL, Zambrano C, Vladimir M. Percepción de cuidado en estudiantes de enfermería: caracterización e impacto para la formación y la visión del ejercicio profesional. Avances en Enfermería. 2008; 26(1).

23. Landeros OE. Identificación de los patrones de conocimiento en un incidente Crítico. Horizonte de Enfermería. 2002; 13:1-7.

24. Muñoz F, Morales J, Torres L. El cine en la enseñanza de estrategias para el afrontamiento de la enfermedad. Enlace Rev Venezolana de Información, Tecnología y Conocimiento. 2009;6 (2): 27-37.

25. Behm K, Et al. Knowledge Development: Patterns and Outcomes. 2006.

26. Muñoz TT, Casique CL. Análisis de la ternura a través de los patrones del conocimiento de Carper: Una experiencia en el cuidado de enfermería. ENE. Rev Enfermería. 2013;7(1):1-7. 
27. Gómez-Palencia I. Técnicas de la narrativa en enfermería, patrones de conocimiento y abordaje teórico. Rev. cienc. Biomed. 2012;3 (1):174-179.

28. Pech GM, Casique CL. Experiencia de una relación afectiva en el cuidado de enfermería, con base en los patrones del conocimiento de Bárbara Carper. 2014; 8 (3).

29. Meza-Benavides M. Construcción del conocimiento en Enfermería: Enfermeros y enfermeras tienen la palabra. Enfermería en Costa Rica. 2006; 27(1):10-16. 
ANEXO: Tabla 3: Resumen de artículos seleccionados

\begin{tabular}{|c|c|c|}
\hline AUTOR, AÑO & DISEÑO & METODOLOGÍA \\
\hline $\begin{array}{l}\text { Gómez RO, } \\
\text { Daza CL, } \\
\text { Zambrano C, } \\
\text { Vladimir M. } \\
\text { (2008). }\end{array}$ & Cuantitativo & $\begin{array}{l}\text { Encuesta a estudiantes de primer semestre de } \\
\text { enfermería para describir la percepción de cuidado } \\
\text { que tienen, abordando los patrones de } \\
\text { conocimiento de Carper. }\end{array}$ \\
\hline $\begin{array}{l}\text { Muñoz, TT, } \\
\text { Casique CL. } \\
\text { (2013). }\end{array}$ & Cualitativo & $\begin{array}{l}\text { Narrativa de una experiencia de cuidado en } \\
\text { pediátrico con secuelas neurológicas y motoras, } \\
\text { involucrando el sentimiento de "ternura" en la } \\
\text { relación enfermera-paciente. }\end{array}$ \\
\hline $\begin{array}{l}\text { Gómez-Palencia I. } \\
\text { (2012). }\end{array}$ & Cualitativo & $\begin{array}{l}\text { Técnica narrativa, en paciente con enfermedad } \\
\text { cardiovascular en servicio de urgencias empleando } \\
\text { los patrones de conocimiento. }\end{array}$ \\
\hline $\begin{array}{l}\text { Bautista EG. } \\
\text { (2015). }\end{array}$ & $\begin{array}{l}\text { Cuantitativo } \\
\text { Descriptivo, } \\
\text { Transversal }\end{array}$ & $\begin{array}{l}\text { Identificó el conocimiento e importancia } \\
\text { gue los estudiantes de enfermería tienen sobre el } \\
\text { consentimiento informado aplicado a los actos } \\
\text { propios del cuidado de enfermería, mediante la } \\
\text { aplicación los patrones del conocimiento } \\
\text { en enfermería. }\end{array}$ \\
\hline $\begin{array}{l}\text { Muñoz F, } \\
\text { Morales J, } \\
\text { Torres L. (2009). }\end{array}$ & $\begin{array}{l}\text { Reflexivo } \\
\text { Revisión }\end{array}$ & $\begin{array}{l}\text { Exponen la experiencia en la utilización de material } \\
\text { cinematográfico en una actividad académica para la } \\
\text { mejora en la implementación de la metodología de } \\
\text { cuidados y las estrategias } \\
\text { gue se utilizaron para la integración de contenidos } \\
\text { teóricos y prácticos. Con el fin de integrar todos } \\
\text { los patrones de conocimiento de Carper como } \\
\text { propuesta educativa. }\end{array}$ \\
\hline Briñez AK. (2014). & Cualitativo & $\begin{array}{l}\text { Narrativa de situación de cuidado para analizar los } \\
\text { patrones de conocimiento en mujer adulta } \\
\text { cuidadora de padre con amputación de origen } \\
\text { diabético, en ámbito extra-hospitalario. }\end{array}$ \\
\hline $\begin{array}{l}\text { Pech GM, } \\
\text { Casique CL. } \\
\text { (2014). }\end{array}$ & Cualitativo & $\begin{array}{l}\text { Narrativa de experiencia sobre afecto como } \\
\text { sentimiento, para identificar la creación una } \\
\text { relación afectiva entre una persona adulto mayor } \\
\text { con Alzheimer que vivía en un asilo y la enfermera. }\end{array}$ \\
\hline $\begin{array}{l}\text { Hernández D. } \\
\text { (2013). }\end{array}$ & Cualitativo & $\begin{array}{l}\text { Narrativa de la experiencia de cuidado en paciente } \\
\text { adulto, mayor crónico oncológico, como "Situación } \\
\text { de enfermería". }\end{array}$ \\
\hline Espitia CL. (2009) & $\begin{array}{l}\text { Reflexión } \\
\text { Análisis }\end{array}$ & $\begin{array}{l}\text { Análisis del cuidado de enfermería en el ámbito de } \\
\text { la cirugía plástica a partir de los patrones de } \\
\text { conocimiento para mostrar cómo a través de éstos } \\
\text { se expresa, desarrolla y sustenta la práctica del } \\
\text { cuidado. }\end{array}$ \\
\hline
\end{tabular}

Fuente: Elaboración propia (2017) 